\title{
Article \\ Solving Bi-Matrix Games Based on Fuzzy Payoffs via Utilizing the Interval Value Function Method
}

\author{
Kaisheng Liu * and Yumei Xing \\ School of Mathematics and Statistics, Tianshui Normal University, Tianshui 741001, China; x_ym2015@163.com \\ * Correspondence: 1ks2999@163.com; Tel.: +86-138-3081-4068
}

Received: 26 March 2019; Accepted: 20 May 2019; Published: 24 May 2019

check for updates

\begin{abstract}
In this article, we introduce a model of bi-matrix games based on crisp parametric payoffs via utilizing the method of interval value function. Then, we get that equilibrium solutions of bi-matrix games on the basis of fuzzy payoffs and equilibrium solutions of the game model are of equal value. Furthermore, it is concluded that equilibrium solutions of the game can be converted to optimal solutions of discrete nonlinear optimization problems with parameters. Lastly, the proposed methodology is illustrated by an example.
\end{abstract}

Keywords: fuzzy bi-matrix games; equilibrium solutions; non-linear optimization problems; interval value function methods

\section{Introduction}

In various areas of the real world, the relations between the interests of two players are very complex, because matrix game has some limitations in practical applications. On this basis, the theory of bi-matrix game is developed. In recent years, bi-matrix game theory has been extensively used in machine learning, complex networks, natural science, social science and military science. Hence, more and more scholars pay attention to bi-matrix game problems.

In 1965, Zadeh [1] introduced the concept of fuzzy sets. Subsequently, the concept of fuzzy numbers is put forward [2-4]. These theories have been widely studied in the theory of bi-matrix game [5-14]. For example, a model of interval bi-matrix games is discussed by Hladik [15]. They obtained the conclusion that solutions of this model are equal to optimal solutions of crisp nonlinear optimization problems based on parameters. Fei et al. [6] utilized the method of bilinear programming models to deal with the interval bi-matrix game problem. A model of bi-matrix game on account of fuzzy payoffs is discussed by Maeda [9]. At the same time, they confirmed the existence of Nash equilibrium in the theory of fuzzy bi-matrix game. Moreover, Vidyottama et al. [12] disposed the model of bi-matrix game based on fuzzy goal by taking advantage of a crisp non-linear programming method. Taking elicitation from [9,12], a bi-matrix game model on the basis of fuzzy payoffs and fuzzy goals is put forward in [13]. By using the method of fuzzy relations, they obtained solutions of this model. Recently, Nayak et al. [11] studied a model of bi-matrix game with goals in the context of intuitionistic fuzzy numbers. Whereafter, through utilizing bilinear programming method and defuzzification ranking method, $\mathrm{Li}$ [8] solved the model of bi-matrix game based on payoffs in the context of intuitionistic fuzzy numbers. On the basis of $[5,6,8]$, we will introduce a non-linear optimization method for a model of bi-matrix games based on crisp parametric payoffs by making use of the method of interval value function in this article.

This article is arranged as follows: Section 2 describes some necessary fundamental knowledge about fuzzy numbers and crisp single objective bi-matrix games. Section 3 presents a model of bi-matrix games based on crisp parametric payoffs via utilizing the method of interval value function. Then, we get that equilibrium solutions of bi-matrix games on the basis of fuzzy payoffs and equilibrium 
solutions of the game model are of equal value. Furthermore, We conclude that equilibrium solutions of the model can converted into optimal solutions of discrete nonlinear programming problems with parameters. Section 4 gives an example problem related to bi-matrix game on the basis of fuzzy payoffs. Section 5 summarizes the whole paper.

\section{Preliminaries}

In the following, we first give the definition of fuzzy number and its related conclusions.

$\mathcal{K}_{C}$ denotes the family of all bounded closed intervals in $\mathbb{R}[16]$, in other words,

$$
\mathcal{K}_{C}=\left\{\left[a_{L}, a_{R}\right] \mid a_{L}, a_{R} \in \mathbb{R} \text { and } a_{L} \leq a_{R}\right\} .
$$

A fuzzy set $\widetilde{x}$ of $\mathbb{R}$ is characterized by a membership function $\mu_{\widetilde{x}}: \mathbb{R} \rightarrow[0,1]$. For each $\widetilde{x}$ and any $\alpha \in(0,1],[\widetilde{x}]^{\alpha}=\left\{x \in \mathbb{R}: \mu_{\widetilde{x}}(x) \geq \alpha\right\}$ denotes a $\alpha$-level set. the set $[\widetilde{x}]^{0}$ is defined by $[\widetilde{x}]^{0}=\overline{\bigcup_{\alpha \in(0,1]}[\widetilde{x}]^{\alpha}}$, where $\bar{A}$ denotes the closure of a crisp set $A$. For all $\alpha \in[0,1]$, a fuzzy number $\widetilde{x}$ is a fuzzy set with non-empty bounded closed level sets $[\widetilde{x}]^{\alpha}=\left[\widetilde{x}_{L}(\alpha), \widetilde{x}_{R}(\alpha)\right]$. Where $\left[\widetilde{x}_{L}(\alpha), \widetilde{x}_{R}(\alpha)\right]$ denotes a closed interval with the left end point $\widetilde{x}_{L}(\alpha)$ and the right end point $\widetilde{x}_{R}(\alpha)$ [17]. $\mathscr{F}$ denotes the family of all fuzzy numbers.

Definition 1 ([18]). Let's assume that $\widetilde{x}$ and $\widetilde{y}$ are two fuzzy numbers. Thus, $\widetilde{x} \preceq \widetilde{y}$ if and only if $[u]^{\alpha}=\left[u_{L}(\alpha)\right.$, $\left.u_{R}(\alpha)\right] \leq[v]^{\alpha}=\left[u_{L}(\alpha), u_{R}(\alpha)\right]$ for each $\alpha \in[0,1]$, where $[u]^{\alpha} \leq[v]^{\alpha}$ if and only if $u_{L}(\alpha) \leq v_{L}(\alpha)$ and $u_{R}(\alpha) \leq v_{R}(\alpha)$.

In $[19,20]$, the order relation $\preceq$ is reflexive and transitive. Furthermore, in the case of $\preceq$, any two fuzzy numbers of $\mathscr{F}$ are comparable.

Definition 2 ([1]). Supposed that $\widetilde{x}$ is a fuzzy number, If $u_{\tilde{x}}(x)$ of $\widetilde{x}$ satisfy the following requirement

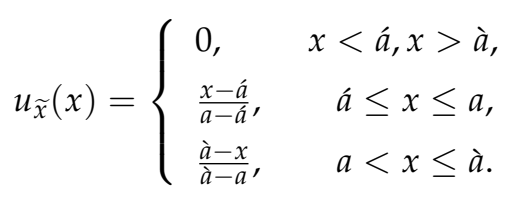

Then, $\widetilde{x}$ is called a triangular fuzzy number, and $\widetilde{x}$ is denoted by $\widetilde{x}=(a, a, a)$.

Moreover, the $\alpha$-level set of $\widetilde{x}=(a, a, \grave{a})$ is defined by [1]

$$
[\widetilde{x}]^{\alpha}=\left[\widetilde{x}_{L}(\alpha), \widetilde{x}_{R}(\alpha)\right]=[(a-\grave{a}) \alpha+\grave{a},-(\grave{a}-a) \alpha+\grave{a}], \alpha \in(0,1] .
$$

Definition 3 ([21]). Suppose that $x_{i} \geq 0(i=1,2, \cdots, n)$ are real numbers and $\widetilde{a}_{i}$ are fuzzy numbers. Thus, $\sum_{i=1}^{n} \widetilde{a}_{i} x_{i}$ also a fuzzy number.

Next, we describe the (discrete) bi-matrix game model of two players and its related knowledge in $[5,22]$.

Definition 4 ([5]). The mixed strategy set of player I is defined as

$$
S^{m}=\left\{x=\left(x_{1}, x_{2}, \cdots, x_{m}\right)^{T} \in \mathbb{R}^{m} \mid \sum_{i=1}^{m} x_{i}=1, x_{i} \geq 0, i=1,2, \cdots, m .\right\}
$$


Analogously, the mixed strategy set of player II is defined as

$$
S^{n}=\left\{y=\left(y_{1}, y_{2}, \cdots, y_{n}\right)^{T} \in \mathbb{R}^{n} \mid \sum_{j=1}^{n} y_{j}=1, y_{j} \geq 0, j=1,2, \cdots, n .\right\},
$$

where $x^{T}$ denotes the transposition of $x, \mathbb{R}^{m}$ denotes $m$-dimensional Euclidean space and $\mathbb{R}^{n}$ denotes n-dimensional Euclidean space.

In terms of bi-matrix game theory, (discrete) payoff matrices are described as [5,22]

$$
A=\left(\begin{array}{ccc}
a_{11} & \cdots & a_{1 n} \\
\vdots & \ddots & \vdots \\
a_{m 1} & \cdots & a_{m n}
\end{array}\right)
$$

and

$$
B=\left(\begin{array}{ccc}
b_{11} & \cdots & b_{1 n} \\
\vdots & \ddots & \vdots \\
b_{m 1} & \cdots & b_{m n}
\end{array}\right)
$$

respectively. Suppose that the player $I$ and player $I I$ are maximized players. A model of bi-matrix game $(B G)$ in [5] is considered as

$$
B G=\left(S^{m}, S^{n}, A, B\right)
$$

Definition 5 ([5,22]). Let's say that $A$ is the payoff matrix for player I. When player I chooses $x \in S^{m}$ and player II chooses $y \in S^{n}$. Then, the expected payoff of player I is defined as

$$
E(x, y, A)=x^{T} A y=\sum_{i=1}^{m} \sum_{j=1}^{n} a_{i j} x_{i} y_{j}
$$

Analogously, let's say that B is the payoff matrix for player II. Then, the expected payoff of player II is defined as

$$
E(x, y, B)=x^{T} B y=\sum_{i=1}^{m} \sum_{j=1}^{n} b_{i j} x_{i} y_{j}
$$

Definition $6([5,6])$. Suppose $(x, y) \in S^{m} \times S^{n}$. If $x \in S^{m}$ and $y \in S^{n}$ satisfy two requirements

$$
\begin{aligned}
& x^{T} A y^{*} \leq\left(x^{*}\right)^{T} A y^{*}, \forall x \in S^{m}, \\
& \left(x^{*}\right)^{T} B y \leq\left(x^{*}\right)^{T} B y^{*}, \forall y \in S^{n} .
\end{aligned}
$$

Thus, $\left(x^{*}, y^{*}\right) \in S^{m} \times S^{n}$ is called an equilibrium solution of $(B G)$

Theorem 1 ([5,6]). Let's say that we have a game $(B G)$. For any $(x, y) \in S^{m} \times S^{n},(x, y) \in S^{m} \times S^{n}\left(x^{*}, y^{*}\right)$ is an equilibrium solution of $(B G)$ if and only if it is an optimal solution of (discrete) nonlinear programming problems (NLP1). 
$(N L P 1)$

$$
\begin{aligned}
& \max \quad\left\{x^{T} A y+x^{T} B y-u-v\right\} \\
& \text { subject to } \quad \sum_{j=1}^{n} a_{i j} y_{j} \leq u(i=1,2, \cdots, m), \\
& \sum_{i=1}^{m} b_{i j} x_{i} \leq v, \quad(j=1,2, \cdots, n), \\
& \sum_{i=1}^{m} x_{i}=1, \\
& \sum_{j=1}^{n} y_{j}=1, \\
& x_{i} \geq 0, y_{j} \geq 0, \\
& u, v \text { unrestricted in sign. }
\end{aligned}
$$

Note that discrete payoff matrices in bi-matrix game theory mean payoff matrices in the classical (i.e., traditional) bi-matrix game theory. The discrete nonlinear programming problem means the classical (i.e., traditional) nonlinear programming problem. So, "discrete" words in this context can be omitted.

\section{A Bi-Matrix Game with Crisp Parametric Payoffs by Interval Value Function Method}

In this section, we will establish the model of bi-matrix games on the basis of crisp parametric payoffs via utilizing the method of interval value function. Suppose that $S^{m}$ and $S^{n}$ are given in the second part. Let's say that all elements of $\widetilde{A}$ and $\widetilde{B}$ are fuzzy numbers. First, the bi-matrix game on the basis of fuzzy payoffs [5], described by BGFP, can be taken as

$$
B G F P=\left(S^{m}, S^{n}, \widetilde{A}, \widetilde{B}\right) .
$$

Definition 7. Let's say that $\widetilde{A}$ is a fuzzy payoff matrix for player I. When player I chooses $x \in S^{m}$ and player II chooses $y \in S^{n}$, then, the fuzzy expected payoff of player I is defined as

$$
E(x, y, \widetilde{A})=x^{T} \widetilde{A} y=\sum_{i=1}^{m} \sum_{j=1}^{n} \widetilde{a}_{i j} x_{i} y_{j}
$$

Analogously, let's say that $\widetilde{B}$ is a fuzzy payoff matrix for player II. Then, the fuzzy expected payoff of player II is defined as

$$
E(x, y, \widetilde{B})=x^{T} \widetilde{B} y=\sum_{i=1}^{m} \sum_{j=1}^{n} \widetilde{b}_{i j} x_{i} y_{j}
$$

Definition 8. Suppose $(x, y) \in S^{m} \times S^{n}$. If $x \in S^{m}$ and $y \in S^{n}$ satisfy two requirements

$$
\begin{aligned}
& x^{T} \widetilde{A} y^{*} \preceq\left(x^{*}\right)^{T} \widetilde{A} y^{*}, \forall x \in S^{m}, \\
& \left(x^{*}\right)^{T} \widetilde{B} y \preceq\left(x^{*}\right)^{T} \widetilde{B} y^{*}, \forall y \in S^{n} .
\end{aligned}
$$

Thus, $\left(x^{*}, y^{*}\right) \in S^{m} \times S^{n}$ is called an equilibrium solution of $(B G F P)$.

So as to get equilibrium solutions of $(B G F P)$, and then, we will construct crisp parametric payoff matrices of player I and player II via utilizing the method of interval value function as follows

$$
I(\widetilde{A})=\left(\begin{array}{ccc}
{\left[\left(\widetilde{a}_{11}\right)_{L}(\alpha),\left(\widetilde{a}_{11}\right)_{R}(\alpha)\right]} & \cdots & {\left[\left(\widetilde{a}_{1 n}\right)_{L}(\alpha),\left(\widetilde{a}_{1 n}\right)_{R}(\alpha)\right]} \\
\vdots & \ddots & \vdots \\
{\left[\left(\widetilde{a}_{m 1}\right)_{L}(\alpha),\left(\widetilde{a}_{m 1}\right)_{R}(\alpha)\right]} & \cdots & {\left[\left(\widetilde{a}_{m n}\right)_{L}(\alpha),\left(\widetilde{a}_{m n}\right)_{R}(\alpha)\right]}
\end{array}\right)
$$


and

$$
I(\widetilde{B})=\left(\begin{array}{ccc}
{\left[\left(\widetilde{b}_{11}\right)_{L}(\alpha),\left(\widetilde{b}_{11}\right)_{R}(\alpha)\right]} & \cdots & {\left[\left(\widetilde{b}_{1 n}\right)_{L}(\alpha),\left(\widetilde{b}_{1 n}\right)_{R}(\alpha)\right]} \\
\vdots & \ddots & \vdots \\
{\left[\left(\widetilde{b}_{m 1}\right)_{L}(\alpha),\left(\widetilde{b}_{m 1}\right)_{R}(\alpha)\right]} & \cdots & {\left[\left(\widetilde{b}_{m n}\right)_{L}(\alpha),\left(\widetilde{b}_{m n}\right)_{R}(\alpha)\right]}
\end{array}\right)
$$

Hence, we obtain that the model of bi-matrix games on the basis of crisp parametric payoffs via utilizing the method of interval value function, denoted by BGIVFP, can be presented as

$$
B G I V F P=\left(S^{m}, S^{n}, I(\widetilde{A}), I(\widetilde{B})\right) .
$$

Next, we give an equivalence-defining statement of equilibrium solutions of (BGFP).

Definition 9. Suppose $(x, y) \in S^{m} \times S^{n}, \alpha \in[0,1]$. If $x \in S^{m}$ and $y \in S^{n}$ satisfy two requirements

$$
\begin{aligned}
& x^{T} I(\widetilde{A}) y^{*} \preceq\left(x^{*}\right)^{T} I(\widetilde{A}) y^{*}, \forall x \in S^{m}, \\
& \left(x^{*}\right)^{T} I(\widetilde{B}) y \preceq\left(x^{*}\right)^{T} I(\widetilde{B}) y^{*}, \forall y \in S^{n} .
\end{aligned}
$$

So, $\left(x^{*}, y^{*}\right) \in S^{m} \times S^{n}$ is called an equilibrium solution of $(B G I V F P)$.

Note that Definition 8 is equal to Definition 9 according to the Definitions 1 and 3.

As far as the above discussion is concerned, we consider a fuzzy bi-matrix game (BGFP). Taking $\left(\widetilde{a}_{i j}, \widetilde{b}_{i j}\right) \in \widetilde{A}_{i j} \times \widetilde{B}_{i j}(i=1,2, \cdots, m ; j=1,2, \cdots, n)$, by Definition 8 , it is obvious that the value of player $I$ is a function of $\widetilde{a}_{i j}$ and the value of player $I I$ is a function of $\widetilde{b}_{i j}$. In other words, the value $u$ of player $I$ is a function of values $\widetilde{a}_{i j}$, denoted by $u=u\left(\widetilde{a}_{i j}\right)$. Meanwhile, $x^{*} \in S^{m}$ of player $I$ is also a function of values $\widetilde{a}_{i j}$, denoted by $x^{*}=x^{*}\left(\widetilde{a}_{i j}\right)$. Analogously, the value $v$ of player $I I$ is a function of values $\widetilde{b}_{i j}$, denoted by $v=v\left(\widetilde{b}_{i j}\right)$. At the same time, $y^{*} \in S^{n}$ of player II is also a function of values $\widetilde{b}_{i j}$, denoted by $y^{*}=y^{*}\left(\widetilde{b}_{i j}\right)$.

Theorem 2. Let's say that we have a game $(B G F P)$, if $\left(\widetilde{a}_{i j}, \widetilde{b}_{i j}\right) \in \widetilde{A}_{i j} \times \widetilde{B}_{i j}(i=1,2, \cdots, m ; j=1,2, \cdots, n)$, then $u=u\left(\widetilde{a}_{i j}\right)$ and $v=v\left(\widetilde{b}_{i j}\right)$ of player I and player II are monotonic nondecreasing functions.

Proof. For any a given game $(B G F P)$ model, $\left(\widetilde{a}_{i j}, \widetilde{b}_{i j}\right) \in \widetilde{A}_{i j} \times \widetilde{B}_{i j}$ and $\left(\widetilde{a}_{i j}, \widetilde{b}_{i j}^{\prime}\right) \in \widetilde{A}_{i j} \times \widetilde{B}_{i j}$. In terms of mixed strategies, we assume that $\left(x^{*}, y^{*}\right)$ and $\left(\left(x^{*}\right)^{\prime},\left(y^{*}\right)^{\prime}\right)$ are respective equilibrium solutions of fuzzy bi-matrix games on the basis of fuzzy payoffs $\widetilde{A}_{i j} \times \widetilde{B}_{i j}$ and $\widetilde{A}_{i j}^{\prime} \times \widetilde{B}_{i j}^{\prime}$, where $\widetilde{A}_{i j}=\left(\widetilde{a}_{i j}\right)_{m \times n}$, $\widetilde{B}_{i j}=\left(\widetilde{b}_{i j}\right)_{m \times n}, \widetilde{A}_{i j}^{\prime}=\left(\widetilde{a}_{i j}^{\prime}\right)_{m \times n}$ and $\widetilde{B}_{i j}^{\prime}=\left(\widetilde{b}_{i j}^{\prime}\right)_{m \times n}$. If $\left(\widetilde{a}_{i j}, \widetilde{b}_{i j}\right) \preceq\left(\widetilde{a}_{i j}^{\prime} \widetilde{b}_{i j}^{\prime}\right)$, by Definition 1 , we have $\left[\widetilde{a}_{i j}\right]^{\alpha} \leq\left[\widetilde{a}_{i j}\right]^{\alpha}$ and $\left[\widetilde{b}_{i j}\right]^{\alpha} \leq\left[\widetilde{b}_{i j}^{\prime}\right]^{\alpha}$. In other words,

$$
\left(\widetilde{a}_{i j}\right)_{L}(\alpha) \leq\left(\widetilde{a}_{i j}\right)_{L}(\alpha), \quad\left(\widetilde{a}_{i j}\right)_{R}(\alpha) \leq\left(\widetilde{a}_{i j}\right)_{R}(\alpha),
$$

and

$$
\left(\widetilde{b}_{i j}\right)_{L}(\alpha) \leq\left(\widetilde{b}_{i j}^{\prime}\right)_{L}(\alpha), \quad\left(\widetilde{b}_{i j}\right)_{R}(\alpha) \leq\left(\widetilde{b}_{i j}^{\prime}\right)_{R}(\alpha)
$$

Thus, we have

$$
\begin{aligned}
& \sum_{i=1}^{m} \sum_{j=1}^{n}\left(x_{i}\right)^{*}\left(\widetilde{a}_{i j}\right)_{L}(\alpha)\left(y_{j}\right)^{*} \leq \sum_{i=1}^{m} \sum_{j=1}^{n}\left(x_{i}\right)^{*}\left(\widetilde{a}_{i j}\right)_{L}(\alpha)\left(y_{j}\right)^{*}, \\
& \sum_{i=1}^{m} \sum_{j=1}^{n}\left(x_{i}\right)^{*}\left(\widetilde{a}_{i j}\right)_{R}(\alpha)\left(y_{j}\right)^{*} \leq \sum_{i=1}^{m} \sum_{j=1}^{n}\left(x_{i}\right)^{*}\left(\widetilde{a}_{i j}\right)_{R}(\alpha)\left(y_{j}\right)^{*},
\end{aligned}
$$




$$
\sum_{i=1}^{m} \sum_{j=1}^{n}\left(x_{i}\right)^{*}\left(\widetilde{b}_{i j}\right)_{L}(\alpha)\left(y_{j}\right)^{*} \leq \sum_{i=1}^{m} \sum_{j=1}^{n}\left(x_{i}\right)^{*}\left(\widetilde{b}_{i j}^{\prime}\right)_{L}(\alpha)\left(y_{j}\right)^{*},
$$

and

$$
\sum_{i=1}^{m} \sum_{j=1}^{n}\left(x_{i}\right)^{*}\left(\widetilde{b}_{i j}\right)_{R}(\alpha)\left(y_{j}\right)^{*} \leq \sum_{i=1}^{m} \sum_{j=1}^{n}\left(x_{i}\right)^{*}\left(\widetilde{b}_{i j}^{\prime}\right)_{R}(\alpha)\left(y_{j}\right)^{*} .
$$

That is to say,

$$
\sum_{i=1}^{m} \sum_{j=1}^{n}\left(x_{i}\right)^{*} \widetilde{a}_{i j}\left(y_{j}\right)^{*} \preceq \sum_{i=1}^{m} \sum_{j=1}^{n}\left(x_{i}\right)^{*} \widetilde{a}_{i j}\left(y_{j}\right)^{*}
$$

and

$$
\sum_{i=1}^{m} \sum_{j=1}^{n}\left(x_{i}\right)^{*} \widetilde{b}_{i j}\left(y_{j}\right)^{*} \preceq \sum_{i=1}^{m} \sum_{j=1}^{n}\left(x_{i}\right)^{*} \widetilde{b}_{i j}^{\prime}\left(y_{j}\right)^{*}
$$

Since in terms of mixed strategies, $\left(\left(x^{*}\right)^{\prime},\left(y^{*}\right)^{\prime}\right)$ is an equilibrium solution of fuzzy bi-matrix game based on fuzzy payoffs $\widetilde{A}_{i j}^{\prime} \times \widetilde{B}_{i j}^{\prime}$, we get

$$
\sum_{i=1}^{m} \sum_{j=1}^{n}\left(x_{i}\right)^{*} \widetilde{a}_{i j}\left(y_{j}\right)^{*} \preceq \sum_{i=1}^{m} \sum_{j=1}^{n}\left(\left(x_{i}\right)^{*}\right)^{\prime} \widetilde{a}_{i j}^{\prime}\left(\left(y_{j}\right)^{*}\right)^{\prime},
$$

and

$$
\sum_{i=1}^{m} \sum_{j=1}^{n}\left(x_{i}\right)^{*} \widetilde{b}_{i j}^{\prime}\left(y_{j}\right)^{*} \preceq \sum_{i=1}^{m} \sum_{j=1}^{n}\left(\left(x_{i}\right)^{*}\right)^{\prime} \widetilde{b}_{i j}^{\prime}\left(\left(y_{j}\right)^{*}\right)^{\prime},
$$

Therefore, combining with (19)-(22), we get

$$
\sum_{i=1}^{m} \sum_{j=1}^{n}\left(x_{i}\right)^{*} \widetilde{a}_{i j}\left(y_{j}\right)^{*} \preceq \sum_{i=1}^{m} \sum_{j=1}^{n}\left(\left(x_{i}\right)^{*}\right)^{\prime} \widetilde{a}_{i j}\left(\left(y_{j}\right)^{*}\right)^{\prime},
$$

and

$$
\sum_{i=1}^{m} \sum_{j=1}^{n}\left(x_{i}\right)^{*} \widetilde{b}_{i j}\left(y_{j}\right)^{*} \preceq \sum_{i=1}^{m} \sum_{j=1}^{n}\left(\left(x_{i}\right)^{*}\right)^{\prime} \widetilde{b}_{i j}^{\prime}\left(\left(y_{j}\right)^{*}\right)^{\prime},
$$

That is,

$$
\left(x^{*}\right)^{T} \widetilde{A} y^{*} \preceq\left(\left(x^{*}\right)^{\prime}\right)^{T} \widetilde{A}^{\prime}\left(y^{*}\right)^{\prime},
$$

and

$$
\left(x^{*}\right)^{T} \widetilde{B} y^{*} \preceq\left(\left(x^{*}\right)^{\prime}\right)^{T} \widetilde{B}^{\prime}\left(y^{*}\right)^{\prime} .
$$

Then $u=u\left(\widetilde{a}_{i j}\right)$ and $v=v\left(\widetilde{b}_{i j}\right)$ of player $I$ and player $I I$ are monotonic nondecreasing functions.

Inspired by [6,23], the values of two players in terms of game BGIVFP model are closed interval, denoted by $[\underline{u}(\alpha), \bar{u}(\alpha)]=\left[u\left(\left(\widetilde{a}_{i j}\right)_{L}(\alpha)\right), u\left(\left(\widetilde{a}_{i j}\right)_{R}(\alpha)\right)\right]$ and $[\underline{v}(\alpha), \bar{v}(\alpha)]=\left[v\left(\left(\widetilde{b}_{i j}\right)_{L}(\alpha)\right), v\left(\left(\widetilde{b}_{i j}\right)_{R}(\alpha)\right)\right]$, respectively, where $\underline{u}(\alpha)=u\left(\left(\widetilde{a}_{i j}\right)_{L}(\alpha)\right), \bar{u}(\alpha)=u\left(\left(\widetilde{a}_{i j}\right)_{R}(\alpha)\right) \underline{v}(\alpha)=v\left(\left(\widetilde{b}_{i j}\right)_{L}(\alpha)\right), \bar{v}(\alpha)=v\left(\left(\widetilde{b}_{i j}\right)_{R}(\alpha)\right)$. By the proof of Theorem 2, $\underline{u}(\alpha)$ and $\underline{v}(\alpha)$ are the lower bounds values of player $I$ and player II. Correspondingly, $\underline{x}(\alpha)=x\left(\left(\widetilde{a}_{i j}\right)_{L}(\alpha)\right)$ and $\underline{y}(\alpha)=y\left(\left(\widetilde{b}_{i j}\right)_{L}(\alpha)\right)$ are equilibrium solutions of player $I$ and player II.

By Theorem 1, we have that $\underline{x}(\alpha)$ and $y(\alpha)$ are equilibrium solutions of player $I$ and player II if and only if, $(\underline{x}(\alpha), \underline{y}(\alpha))$ is an optimal solution of discrete nonlinear programming problems with parameters (NLP2). 


$$
\begin{gathered}
(N L P 2) \max \left\{\sum_{i=1}^{m} \sum_{j=1}^{n} \underline{x}_{i}(\alpha)\left(\widetilde{a}_{i j}\right)_{L}(\alpha) \underline{y}_{j}(\alpha)+\sum_{i=1}^{m} \sum_{j=1}^{n} \underline{x}_{i}(\alpha)\left(\widetilde{b}_{i j}\right)_{L}(\alpha) \underline{y}_{j}(\alpha)-\underline{u}(\alpha)-\underline{v}(\alpha)\right\} \\
\text { subject to } \quad \sum_{j=1}^{n}\left(\widetilde{a}_{i j}\right)_{L}(\alpha) \underline{y}_{j}(\alpha) \leq \underline{u}(\alpha)(i=1,2, \cdots, m), \\
\\
\sum_{i=1}^{m}\left(\widetilde{b}_{i j}\right)_{L}(\alpha) \underline{x}_{i}(\alpha) \leq \underline{v}(\alpha), \quad(j=1,2, \cdots, n), \\
\sum_{i=1}^{m} \underline{x}_{i}=1, \\
\sum_{j=1}^{n} \underline{y}_{j}=1, \\
\underline{x}_{i} \geq 0, \underline{y}_{j} \geq 0, \\
\alpha \in[0,1] .
\end{gathered}
$$

Similarly, by the proof of Theorem $2, \bar{u}(\alpha)$ and $\bar{v}(\alpha)$ are the upper bounds values of player $I$ and player II. Correspondingly, $\bar{x}(\alpha)=x\left(\left(\widetilde{a}_{i j}\right)_{R}(\alpha)\right)$ and $\bar{y}(\alpha)=y\left(\left(\widetilde{b}_{i j}\right)_{R}(\alpha)\right)$ are equilibrium solutions of player $I$ and player $I I$.

By Theorem 1, we have that $\bar{x}(\alpha)$ and $\bar{y}(\alpha)$ are equilibrium solutions of player I and player II if and only if, $(\bar{x}(\alpha), \bar{y}(\alpha))$ is an optimal solution of discrete nonlinear programming problems with parameters (NLP3).

$(N L P 3) \max \left\{\sum_{i=1}^{m} \sum_{j=1}^{n} \bar{x}_{i}(\alpha)\left(\widetilde{a}_{i j}\right)_{R}(\alpha) \bar{y}_{j}(\alpha)+\sum_{i=1}^{m} \sum_{j=1}^{n} \bar{x}_{i}(\alpha)\left(\widetilde{b}_{i j}\right)_{R}(\alpha) \bar{y}_{j}(\alpha)-\bar{u}(\alpha)-\bar{v}(\alpha)\right\}$

$$
\begin{array}{ll}
\text { subject to } & \sum_{j=1}^{n}\left(\widetilde{a}_{i j}\right)_{R}(\alpha) \bar{y}_{j}(\alpha) \leq \bar{u}(\alpha) \quad(i=1,2, \cdots, m), \\
& \sum_{i=1}^{m}\left(\widetilde{b}_{i j}\right)_{R}(\alpha) \bar{x}_{i}(\alpha) \leq \bar{v}(\alpha),(j=1,2, \cdots, n), \\
& \sum_{i=1}^{m} \bar{x}_{i}=1 \\
& \sum_{j=1}^{n} \bar{y}_{j}=1 \\
& \bar{x}_{i} \geq 0, \bar{y}_{j} \geq 0 \\
& \alpha \in[0,1] .
\end{array}
$$

Note that for two-player non-zero-sum (bi-matrix) game with fuzzy payoffs $(B G F P)$, there should be a possibility of multiple equilibrium strategies with each producing different payoffs for the players, and not just one game value.

\section{Example}

To verify the validity of discrete nonlinear programming problems with parameters (NLP2) and (NLP3). Now, we will consider the following example.

Example 1. We consider the case of clothing company I and clothing company II making a decision as to how to occupy a sale target market. Due to the uncertainty and imprecision of information, we cannot use any single value to represent the payoff for any one of the sale planning strategies. Thus, it seems to be more realistic and appropriate for expressing payoffs with fuzzy payoffs. 
Suppose that clothing company I and clothing company II are rational players in the same industry. (Where clothing company I and clothing company II represent player I and player II in the above game $(B G F P)$, respectively.) They will choose optimal strategies to maximize their own profits without cooperation. The clothing company I has two alternative marketing strategies: reducing-price sale $\left(\alpha_{1}\right)$ and regular-price sale $\left(\alpha_{2}\right)$. Similarly, The clothing company II has two alternative marketing strategies: reducing-price sale $\left(\beta_{1}\right)$ and regular-price sale $\left(\beta_{2}\right)$. Then, profits matrices for two clothing companies I and II are considered in the fuzzy payoff matrices as follows

$$
\widetilde{A}=\left(\begin{array}{ll}
(11.75,12.00,12.60) & (16.80,17.00,18.00) \\
(12.50,13.00,13.25) & (15.75,16.00,16.25)
\end{array}\right)
$$

and

$$
\widetilde{B}=\left(\begin{array}{cc}
(10.80,11.00,11.70) & (14.75,15.00,15.45) \\
(9.50,10.00,10.80) & (13.50,14.00,14.55)
\end{array}\right)
$$

respectively. Where $\alpha_{1}$ and $\alpha_{2}$ represent the first row and the second row of $\widetilde{A}$, respectively. $\beta_{1}$ and $\beta_{2}$ represent the first column and the second column of $\widetilde{B}$, respectively. $(11.75,12.00,12.60)$ denotes the profit of the clothing company I and $(10.80,11.00,11.70)$ denotes the profit of the clothing company II if the clothing company I chooses reducing-price sale $\left(\alpha_{1}\right)$ and the clothing company II chooses reducing-price sale $\left(\beta_{1}\right)$. Similarly, other elements in $\widetilde{A}$ and $\widetilde{B}$ can be given.

Because elements of fuzzy payoff matrices for clothing company $I$ and clothing company II are fuzzy numbers and a fuzzy number is a fuzzy set with non-empty bounded closed level sets (closed interval), two clothing companies try to determine the ranges of the expected profits. Combining (1), (11) and (12), we obtain the following crisp parametric profits matrices of clothing company $I$ and clothing company II by taking advantage of the interval value function method

$$
I(\widetilde{A})=\left(\begin{array}{ll}
{[0.25 \alpha+11.75,-0.60 \alpha+12.60]} & {[0.20 \alpha+16.80,-1.00 \alpha+18.00]} \\
{[0.50 \alpha+12.50,-0.25 \alpha+13.25]} & {[0.25 \alpha+15.75,-0.25 \alpha+16.25]}
\end{array}\right)
$$

and

$$
I(\widetilde{B})=\left(\begin{array}{cc}
{[0.20 \alpha+10.80,-0.70 \alpha+11.70]} & {[0.25 \alpha+14.75,-0.45 \alpha+15.45]} \\
{[0.50 \alpha+9.50,-0.80 \alpha+10.80]} & {[0.50 \alpha+13.50,-0.55 \alpha+14.55]}
\end{array}\right)
$$

respectively. Thus now utilizing (27) and (28), we obtain the following problems (NLP4) and (NLP5).

$$
\begin{aligned}
& (N L P 4) \quad \max \quad\left\{(0.45 \alpha+22.55) \underline{x}_{1} \underline{y}_{1}+(\alpha+22) \underline{x}_{2} \underline{y}_{1}+(0.45 \alpha+31.55) \underline{x}_{1} \underline{y}_{2}\right. \\
& \left.+(0.75 \alpha+29.25) \underline{x}_{2} \underline{y}_{2}-\underline{u}(\alpha)-\underline{v}(\alpha)\right\} \\
& \text { subject to } \quad(0.25 \alpha+11.75) \underline{y}_{1}+(0.20 \alpha+16.80) \underline{y}_{2} \leq \underline{u}(\alpha) \text {, } \\
& (0.50 \alpha+12.50) \underline{y}_{1}+(0.25 \alpha+15.75) \underline{y}_{2} \leq \underline{u}(\alpha) \text {, } \\
& (0.20 \alpha+10.80) \underline{x}_{1}+(0.50 \alpha+9.50) \underline{x}_{2} \leq \underline{v}(\alpha), \\
& (0.25 \alpha+14.75) \underline{x}_{1}+(0.50 \alpha+13.50) \underline{x}_{2} \leq \underline{v}(\alpha) \text {, } \\
& \underline{x}_{1}+\underline{x}_{2}=1, \underline{y}_{1}+\underline{y}_{2}=1 \text {, } \\
& \underline{x}_{1} \geq 0, \underline{x}_{2} \geq 0, \underline{y}_{1} \geq 0, \underline{y}_{2} \geq 0 \text {, } \\
& \alpha \in[0,1] \text {. }
\end{aligned}
$$


and

(NLP5)

$$
\begin{aligned}
& \max \left\{(-1.30 \alpha+24.3) \bar{x}_{1} \bar{y}_{1}+(-1.05 \alpha+24.05) \bar{x}_{2} \bar{y}_{1}+(-1.45 \alpha+33.45) \bar{x}_{1} \bar{y}_{2}\right. \\
& \left.+(-0.8 \alpha+30.8) \bar{x}_{2} \bar{y}_{2}-\bar{u}(\alpha)-\bar{v}(\alpha)\right\} \\
& \text { subject to } \quad(-0.60 \alpha+12.60) \bar{y}_{1}+(-1.00 \alpha+18.00) \bar{y}_{2} \leq \bar{u}(\alpha) \text {, } \\
& (-0.25 \alpha+13.25) \bar{y}_{1}+(-0.25 \alpha+16.25) \bar{y}_{2} \leq \bar{u}(\alpha), \\
& (-0.70 \alpha+11.70) \bar{x}_{1}+(-0.80 \alpha+10.80) \bar{x}_{2} \leq \bar{v}(\alpha), \\
& (-0.45 \alpha+15.45) \bar{x}_{1}+(-0.55 \alpha+14.55) \bar{x}_{2} \leq \bar{v}(\alpha), \\
& \bar{x}_{1}+\bar{x}_{2}=1, \bar{y}_{1}+\bar{y}_{2}=1 \text {, } \\
& \bar{x}_{1} \geq 0, \bar{x}_{2} \geq 0, \bar{y}_{1} \geq 0, \bar{y}_{2} \geq 0, \\
& \alpha \in[0,1] \text {. }
\end{aligned}
$$

Utilizing the Lingo software, particularly, let $\alpha^{*}=0.2$, we can get that $\left(\underline{x}^{*}, \underline{y}^{*}, \underline{u}^{*}, \underline{v}^{*}\right)$ and $\left(\bar{x}^{*}, \bar{y}^{*}, \bar{u}^{*}, \bar{v}^{*}\right)$ are optimal solutions of problems (NLP4) and (NLP5), respectively, where $\underline{x}^{*}=$ $\left(\underline{x}_{1}^{*}=0.32, \underline{x}_{2}^{*}=0.68\right), \underline{y}^{*}=\left(\underline{y}_{1}^{*}=0.24, \underline{y}_{2}^{*}=0.76\right), \underline{u}^{*}=\underline{u}\left(\alpha^{*}\right)=15.63, \underline{v}^{*}=\underline{v}\left(\alpha^{*}\right)=11.26$, $\bar{x}^{*}=\left(\bar{x}_{1}^{*}=0.37, \bar{x}_{2}^{*}=0.6 \overline{3}\right), \bar{y}^{*}=\left(\bar{y}_{1}^{*}=0.30, \bar{y}_{2}^{*}=0.70\right), \bar{u}^{*}=\bar{u}\left(\alpha^{*}\right)=16.20, \bar{v}^{*}=\bar{v}\left(\alpha^{*}\right)=14.78$.

Thus, we obtain that $\underline{u}^{*}=15.63$ and $\underline{v}^{*}=11.26$ are the lower bounds values of clothing company $I$ and clothing company II. Correspondingly, $\underline{x}^{*}=\left(\underline{x}_{1}^{*}=0.32, \underline{x}_{2}^{*}=0.68\right)$ and $\underline{y}^{*}=\left(\underline{y}_{1}^{*}=0.24, \underline{y}_{2}^{*}=0.76\right)$ are equilibrium solutions of clothing company $I$ and clothing company II.

Similarly, $\bar{u}^{*}=16.20$ and $\bar{v}^{*}=14.78$ are the upper bounds values of clothing company $I$ and clothing company II. Correspondingly, $\bar{x}^{*}=\left(\bar{x}_{1}^{*}=0.37, \bar{x}_{2}^{*}=0.63\right)$ and $\bar{y}^{*}=\left(\bar{y}_{1}^{*}=0.30, \bar{y}_{2}^{*}=0.70\right)$ are equilibrium solutions of clothing company I and clothing company II. So, let $\alpha^{*}=0.2,\left[\underline{u}^{*}, \bar{u}^{*}\right]=$ $[15.63,16.20]$ denotes expected profit of clothing company $I$ and $\left[\underline{v}^{*}, \bar{v}^{*}\right]=[11.26,14.78]$ expected profit of clothing company II. That is to say, expected profits of clothing company I and clothing company II belong to some ranges.

Similarly, for other values of $\alpha \in(0,1]$, we also get corresponding expected profits of clothing company $I$ and clothing company II. Expected profits of clothing company I and clothing company II belong to some ranges.

\section{Conclusions}

This article has presented a model of bi-matrix game (BGIVFP) based on crisp parametric payoffs via making use of the method of interval value function. Then, we get that equilibrium solutions of bi-matrix games on the basis of fuzzy payoffs and equilibrium solutions of the game (BGIVFP) are of equal value. It is concluded that equilibrium solutions of the game (BGIVFP) can be converted to optimal solutions of discrete nonlinear programming problems with parameters (NLP3). At present, machine learning and complex neural networks have been widely researched by many scholars. Hence, we will apply the relevant theories in this paper to machine learning and complex neural networks.

Author Contributions: K.L. conceived of the main thoughts; Y.X. drafted and revised the paper; all authors read and approved the final manuscript.

Acknowledgments: The authors would like to thank all of the reviewers for their valuable comments.

Conflicts of Interest: The authors declare no conflict of interest.

\section{References}

1. Zadeh, L.A. Fuzzy sets. Inf. Control 1965, 8, 338-353. [CrossRef]

2. Zadeh, L.A. The concept of a linguistic variable and its application to approximate reasoning-I. Inf. Sci. 1975, 8, 199-249. [CrossRef] 
3. Zadeh, L.A. The concept of a linguistic variable and its application to approximate reasoning-II. Inf. Sci. 1975, 8, 301-357. [CrossRef]

4. Zadeh, L.A. The concept of a linguistic variable and its application to approximate reasoning-III. Inf. Sci. 1975, 9, 43-80. [CrossRef]

5. Bector, C.R.; Chandra, S. Fuzzy Mathematical Programming and Fuzzy Matrix Games; Springer: Berlin/Heidelberg, Germany, 2005.

6. Fei, W.; Li, D.F. Bilinear Programming Approach to Solve Interval Bimatrix Games in Tourism Planning Management. Int. J. Fuzzy Syst. 2016, 18, 504-510. [CrossRef]

7. Li, C. Characterization of the Equilibrium Strategy of Fuzzy Bimatrix Games Based on Fuzzy Variables. J. Appl. Math. 2012, 3, 331-353. [CrossRef]

8. Li, D.F. Decision and Game Theory in Management with Intuitionistic Fuzzy Sets. In Studies in Fuzziness and Soft Computing; Springer: Berlin/Heidelberg, Germany, 2014; Volume 308.

9. Maeda, T. On characterization of equilibirum strategy of the bimatrix game with fuzzy payoff. J. Math. Anal. Appl. 2000, 251, 885-896. [CrossRef]

10. Nan, J.X.; Zhang, M.J.; Li, D.F. Intuitionistic fuzzy programming models for matrix games with payoffs of trapezoidal intuitionistic fuzzy numbers. Int. J. Fuzzy Syst. 2014, 16, 444-456.

11. Nayak, P.K.; Pal, M. Bimatrix games with intiutionstic fuzzy goals. Iran. J. Fuzzy Syst. 2010, 7, 65-79.

12. Vidyottama, V.; Chandra S.; Bector, C.R. Bi-matrix game with fuzzy goals and fuzzy payoffs. Fuzzy Optim. Decis. Mak. 2004, 3, 327-344. [CrossRef]

13. Vijay, V.; Mehra, A.; Chandra, S.; Bector, C.R. Fuzzy matrix games via a fuzzy relation approach. Fuzzy Optim. Decis. Mak. 2007, 6, 299-314. [CrossRef]

14. Larbani, M. Solving bi-matrix games with fuzzy payoffs by introducing nature as a third player. Fuzzy Sets Syst. 2009, 160, 657-666. [CrossRef]

15. Hladk, M. Interval valued bimatrix games. Kybernetika Praha 2010, 3, 435-446.

16. Diamond, P.; Kloeden, P. Metric spaces of fuzzy sets. Fuzzy Sets Syst. 1990, 35, 241-249. [CrossRef]

17. Dubois, D.; Prade, H. Fuzzy Sets and Systems-Theory and Application; Academic Press: New York, NY, USA, 1980.

18. Wang, G.X.; Wu, C.X. Directional derivatives and subdifferential of convex fuzzy mappings and application in convex fuzzy programming. Fuzzy Sets Syst. 2003, 138, 559-591. [CrossRef]

19. Syau, Y.R.; Stanley Lee, E. Fuzzy Weirstrass theorem and convex fuzzy mappings. Comput. Math. Appl. 2006, 51, 1741-1750. [CrossRef]

20. Syau, Y.R.; Stanley Lee, E. Preinvexity and F1-convexity of fuzzy mappings through a linear ordering. Comput. Math. Appl. 2006, 51, 405-418. [CrossRef]

21. Li, L.; Liu, S.Y.; Zhang, J.K. On fuzzy generalized convex mappings and optimality conditions for fuzzy weakly univex mappings. Fuzzy Sets Syst. 2015, 280, 107-132. [CrossRef]

22. Basar, T.; Olsder, G.J. Dynamic Noncooperative Game Theory, 2nd ed.; Academic: San Diego, CA, USA, 1995.

23. Davvaz, B.; Khan, A.; Sarmin, N.H. More general forms of interval valued fuzzy filters of ordered semigroups. Int. J. Fuzzy Syst. 2013, 15, 110-126.

(C) 2019 by the authors. Licensee MDPI, Basel, Switzerland. This article is an open access article distributed under the terms and conditions of the Creative Commons Attribution (CC BY) license (http://creativecommons.org/licenses/by/4.0/). 